\title{
Generalized vortex-model for the inverse cascade of two-dimensional turbulence
}

\author{
J. Friedrich, 1 and R. Friedrich ${ }^{2}$ \\ ${ }^{1}$ Institute for Theoretical Physics I, Ruhr-University Bochum, \\ Universitätsstr. 150, D-44801 Bochum, Germany \\ ${ }^{2}$ Institute for Theoretical Physics, University of Münster, \\ Wilhelm-Klemm-Str. 9, D-48149 Münster, Germany
}

(Dated: October 1, 2018)

\begin{abstract}
We generalize Kirchhoff's point vortex model of two-dimensional fluid motion to a rotor model which exhibits an inverse cascade by the formation of rotor clusters. A rotor is composed of two vortices with like-signed circulations glued together by an overdamped spring. The model is motivated by a treatment of the vorticity equation representing the vorticity field as a superposition of vortices with elliptic Gaussian shapes of variable widths, augmented by a suitable forcing mechanism. The rotor model opens up the way to discuss the energy transport in the inverse cascade on the basis of dynamical systems theory.

PACS numbers: 47.27.-i 05.40.Fb 05.10.Gg 52.65.Ff
\end{abstract}

\section{INTRODUCTION}

The theoretical treatment of the longstanding problem of turbulent flows [1 4] has to relate dynamical systems theory with non-equilibrium statistical physics [5]. The central notion of physical turbulence theory is the concept of the energy cascade, highlighting the fact that turbulent flows are essentially transport processes of quantities like energy or enstrophy in scale. Although wellestablished theories due to Richardson, Kolmogorov, Onsager, Heisenberg and others (for reviews we refer the reader to [1 3$]$ ) can capture gross features of the cascade process in a phenomenological way, the dynamical aspects are by far less understood, and usually are investigated by direct numerical simulations of the NavierStokes equations.

An exception, in some sense, are inviscid fluid flows in two dimensions. Based on the work of Helmholtz [ [6], it was Kirchhoff [7] who pointed out that the partial differential equation can be reduced to a Hamiltonian system for the locations of point vortices, provided one considers initial conditions where the vorticity is a superposition of delta-distributions (we refer the reader to the works of Aref [8 10] as well as the monographs [11, 12]). Due to Onsager [13] (for a discussion we refer the reader to [14]) a statistical treatment of point vortex dynamics is possible for equilibrium situations because of the Hamiltonian character of the dynamics, provided the ergodic hypothesis holds. Extensions to non-equilibrium situations based on kinetic equations have been pursued, e.g., by Joyce and Montgomery [15], Lundgren and Pointin [16], as well as more recently by Chavanis [17].

The purpose of the present article is to generalize Kirchhoff's point vortex model to a rotor model that exhibits the formation of large-scale vortical structures due to the formation of rotor clusters. The existence of

\footnotetext{
* Electronic mail: jaf@tp1.rub.de
}

such a process in two-dimensional flows where a largescale vorticity field spontaneously emerges from an initially random distribution of vortices was first predicted by Kraichnan [18] and is termed an inverse cascade. Thereby, the energy that is injected into the small scales is transfered to larger scales, whereas the enstrophy follows a direct cascade from large to small scales. It was also Kraichnan [19], who gave an intuitive explanation of the possible mechanism of the cascade: He considered a small-scale axisymmetric vortical structure that is exposed to a large-scale strain field. Eventually, the vortex is elongated along the stretching direction of the strain, i.e. to a first approximation drawn out into an elliptical structure. This thinning mechanism induces relative motions between vortices that have been deformed under their mutual strain, which leads to a decrease of the kinetic energy of the small-scale motion and consequently to an energy transfer upscale. More recently, it has been pointed out numerically and experimentally by Chen et al. 20] that the effect of vortex thinning is indeed an important feature of the inverse cascade. An appropriate vortex model for the inverse cascade therefore has to provide a mechanism similar to that identified in [20].

Although, several point vortex models have been known for a long time to form large-scale vortical structures from an initially random distribution of point vortices due to the events of vortex merging [21, 22] or special forcing mechanisms 23], an explicit inclusion of the concept of vortex thinning never has been taken into account.

In our vortex model, the small-scale vortical structure is represented by a rotor consisting of two point vortices with equal circulation that are glued together by a nonelastic bond. The main observation now is that the two co-rotating point vortices mimic a far-field that is similar to an elliptical vortex, which makes the rotor sensitive to a large-scale strain. The model is motivated by a representation of the vorticity field as a superposition of vortices with elliptical Gaussian shapes along the lines of Melander, Styczek and Zabusky [25]. The 
nonelastic bond in a rotor can be considered as an overdamped spring which models the influence of forcing and viscous damping.

However, the main renewal in this model is not the mechanism of how the energy is injected into the system, but how the energy is transfered upscale due to the strain-induced relative motions between the rotors in the sense of vortex thinning. The efficiency of the cascade in the rotor model is supported by the relatively fast demixing of the system as well as a Kolmogorov constant of $C_{K}=5.89 \pm 0.74$ that is within the range of accepted values [29 33$]$.

This paper is organized as follows: First of all, we consider a decomposition of the vorticity field into localized vortices with different shapes in section II In section III. we make an ansatz for the shapes which corresponds to an elliptical distribution of the vorticity and discuss the interaction of two vortices with like-signed circulation within the point vortex model, the Gaussian vortex model and the elliptical model. It will explicitly be shown that the former two models do not lead to a relative motion between the vortices, and that the thinning mechanism is only taken into account by the elliptical model. A suitable forcing mechanism for the vorticity equation is introduced in section $\mathrm{V}$ and then used within our generalized vortex model, presented in section VI.

\section{DECOMPOSITION OF THE VORTICITY FIELD INTO VORTICES WITH ARBITRARY SHAPES}

As it is known from basic fluid dynamics, the vorticity

$$
\boldsymbol{\omega}(\mathbf{x}, t)=\nabla \times \mathbf{u}(\mathbf{x}, t)
$$

only possesses one component in two-dimensional flows and obeys the evolution equation

$$
\dot{\omega}(\mathbf{x}, t)+\mathbf{u}(\mathbf{x}, t) \cdot \nabla \omega(\mathbf{x}, t)=\nu \nabla^{2} \omega(\mathbf{x}, t)
$$

Here, the advecting velocity field is determined by BiotSavart's law according to

$$
\mathbf{u}(\mathbf{x}, t)=\int \mathrm{d} \mathbf{x}^{\prime} \omega\left(\mathbf{x}^{\prime}, t\right) \mathbf{e}_{z} \times \frac{\mathbf{x}-\mathbf{x}^{\prime}}{2 \pi\left|\mathbf{x}-\mathbf{x}^{\prime}\right|^{2}}
$$

We consider the two-dimensional vorticity equation in Fourier space

$$
\dot{\omega}(\mathbf{k}, t)-i \mathbf{k} \cdot \int \mathrm{d} \mathbf{k}^{\prime} \mathbf{u}\left(\mathbf{k}^{\prime}\right) \omega\left(\mathbf{k}-\mathbf{k}^{\prime}, t\right) \omega\left(\mathbf{k}^{\prime}, t\right)=-\nu k^{2} \omega(\mathbf{k}, t)
$$

with $\mathbf{u}(\mathbf{k})=\frac{i}{4 \pi^{2}}\left[\mathbf{e}_{z} \times \frac{\mathbf{k}^{\prime}}{k^{\prime 2}}\right]$.

In the following the vorticity is decomposed into vortices $\omega_{j}(\mathbf{k}, t)$ with the circulation $\Gamma_{j}$ that are centered at $\mathbf{x}_{j}(t)$ and that possess the shapes $W_{j}(\mathbf{k}, t)$, namely

$$
\omega_{j}(\mathbf{k}, t)=\Gamma_{j} e^{i \mathbf{k} \cdot \mathbf{x}_{j}(t)+W_{j}(\mathbf{k}, t)}
$$

Our ansatz thus reads

$$
\omega(\mathbf{k}, t)=\sum_{j} \omega_{j}(\mathbf{k}, t)=\sum_{j} \Gamma_{j} e^{i \mathbf{k} \cdot \mathbf{x}_{j}(t)+W_{j}(\mathbf{k}, t)}
$$

For $W_{j}(\mathbf{k}, t)=0$, we recover the vorticity field $\omega(\mathbf{x}, t)$ of point vortices

$$
\omega(\mathbf{x}, t)=\sum_{j} \Gamma_{j} \delta\left(\mathbf{x}-\mathbf{x}_{j}(t)\right)
$$

that are located at the positions $\mathbf{x}_{j}(t)$ and that are a solution of the ideal vorticity equation $(\nu=0)$, which conserves the vorticity along a Lagrangian trajectory. Inserting the vorticity field from (7) into Biot-Savart's law (3) immediately yields the evolution equation for the point vortices

$$
\dot{\mathbf{x}}_{j}(t)=\sum_{l} \frac{\Gamma_{l}}{2 \pi} \mathbf{e}_{z} \times \frac{\mathbf{x}_{j}(t)-\mathbf{x}_{l}(t)}{\left|\mathbf{x}_{j}(t)-\mathbf{x}_{l}(t)\right|^{2}}
$$

We now insert our ansatz (6) into the vorticity equation and obtain

$$
\begin{aligned}
& \sum_{j} \Gamma_{j} e^{i \mathbf{k} \cdot \mathbf{x}_{j}+W_{j}(\mathbf{k}, t)}\left[i \mathbf{k} \cdot \dot{\mathbf{x}}_{j}(t)+\dot{W}_{j}(\mathbf{k}, t)+\nu k^{2}\right] \\
= & i \mathbf{k} \cdot \sum_{j, l} \Gamma_{j} \Gamma_{l} \int \mathrm{d} \mathbf{k}^{\prime} \mathbf{u}\left(\mathbf{k}^{\prime}\right) e^{i\left(\mathbf{k}-\mathbf{k}^{\prime}\right) \cdot \mathbf{x}_{\mathbf{j}}+i \mathbf{k}^{\prime} \cdot \mathbf{x}_{l}} \\
& \times e^{W_{j}\left(\mathbf{k}-\mathbf{k}^{\prime}, t\right)+W_{l}\left(\mathbf{k}^{\prime}, t\right)}
\end{aligned}
$$

The left-hand side of this equation contains the sweeping dynamic of the vortices encoded in the temporal change of $\mathbf{x}_{j}(t)$ as well as the temporal change of the shapes $W_{j}(\mathbf{k}, t)$ due to shearing and vorticity. In the inviscid case, the entire dynamic of the $j$-th vortex is determined by the nonlinearity on the right hand side of equation (9) which couples the different Fourier modes of the vortices $l$ as well as the self-interaction term from $j=l$ in a rather complicated manner.

Nevertheless, a separation of the effects becomes possible under the assumption that the overlap of the different vortex structures is negligible, which is valid for widely separated vortices. To this end, we single out the terms in the summations over $j$ and get

$$
\begin{aligned}
& i \mathbf{k} \cdot \dot{\mathbf{x}}_{j}(t)+\dot{W}_{j}(\mathbf{k}, t)+\nu k^{2} \\
= & i \mathbf{k} \cdot \sum_{l} \Gamma_{l} \int \mathrm{d} \mathbf{k}^{\prime} \mathbf{u}\left(\mathbf{k}^{\prime}\right) e^{-i \mathbf{k}^{\prime} \cdot\left(\mathbf{x}_{\mathbf{j}}-\mathbf{x}_{l}\right)} \\
& \times e^{W_{j}\left(\mathbf{k}-\mathbf{k}^{\prime}, t\right)-W_{j}(\mathbf{k}, t)+W_{l}\left(\mathbf{k}^{\prime}, t\right)}
\end{aligned}
$$

The sweeping dynamic can now be defined via the terms in the evolution equation (10) which are proportional to k. This immediately yields the evolution equations for the center of the vortices

$$
\dot{\mathbf{x}}_{j}(t)=\sum_{l} \Gamma_{l} \mathbf{U}_{j l}\left(\mathbf{x}_{j}-\mathbf{x}_{l}\right)
$$


where we have defined the velocity kernels

$$
\mathbf{U}_{j l}(\mathbf{r})=\int \mathrm{d} \mathbf{k}^{\prime} \mathbf{u}\left(\mathbf{k}^{\prime}\right) e^{-i \mathbf{k}^{\prime} \cdot \mathbf{r}} e^{W_{j}\left(-\mathbf{k}^{\prime}, t\right)+W_{l}\left(\mathbf{k}^{\prime}, t\right)}
$$

Inserting the evolution equation of the vortex centers back into (9) yields the evolution equations for the shapes

$$
\begin{aligned}
& \dot{W}_{i}(\mathbf{k}, t)=-\nu k^{2} \\
& +i \mathbf{k} \cdot \sum_{l} \Gamma_{l} \int d \mathbf{k}^{\prime} \mathbf{u}\left(\mathbf{k}^{\prime}\right) e^{-i \mathbf{k}^{\prime} \cdot\left[\mathbf{x}_{i}-\mathbf{x}_{l}\right]} e^{W_{i}\left(-\mathbf{k}^{\prime}, t\right)+W_{l}\left(\mathbf{k}^{\prime}, t\right)} \\
& \times\left[e^{W_{i}\left(\mathbf{k}-\mathbf{k}^{\prime}, t\right)-W_{i}\left(-\mathbf{k}^{\prime}, t\right)-W_{i}(\mathbf{k}, t)}-1\right]
\end{aligned}
$$

Here the sum includes also the self-interaction term with $j=l$. The system of equations (11) and (13) is the extension of the set of evolution equations for the $\delta$-point vortices (7) and takes into account possible changes of the shapes $W_{j}(\mathbf{k}, t)$ of each vortex. It is important to stress that up to now we did not impose any restrictions on the shapes $W_{j}(\mathbf{k}, t)$.

\section{APPROXIMATION VIA VORTICES WITH ELLIPTICAL SHAPES}

The vorticity of an elliptical vortex with major and minor semi-axes $\mathbf{a}$ and $\mathbf{b}$ can be written according to

$$
\omega(\mathbf{x}, t)=\frac{\Gamma}{\pi} e^{-\mathbf{x} C \mathbf{x}}
$$

where $C=\mathbf{a} \mathbf{a}+\mathbf{b b}$ is the symmetric matrix of the dyadic products of the semi axes. A rotation of the coordinate system then turns (14) into

$$
\omega(\mathbf{x}, t)=\frac{\Gamma}{\pi} e^{-\left(q^{-2} x^{2}+q^{2} y^{2}\right)}
$$

where $q$ is the is the ratio of the major to the minor semi axes. The vorticity in Fourier space thus reads

$$
\omega(\mathbf{k}, t)=\Gamma e^{-\left(q^{2} k_{x}^{2}+q^{-2} k_{y}^{2}\right)}
$$

which again corresponds to an elliptical distribution of the vorticity. An elliptical representation of the shapes can thus be obtained via the approximation

$$
W_{j}(\mathbf{k}, t) \approx-\frac{1}{2} \mathbf{k} C_{j}(t) \mathbf{k}
$$

with the symmetric matrix $C_{j}(t)$. In approximating the last term on the right-hand side of equation (13) by

$$
\begin{aligned}
& e^{W_{j}\left(\mathbf{k}-\mathbf{k}^{\prime}, t\right)-W_{j}\left(-\mathbf{k}^{\prime}, t\right)-W_{j}(\mathbf{k}, t)}-1 \\
\approx & -\frac{1}{2}\left[\mathbf{k} C_{j}(t) \mathbf{k}^{\prime}+\mathbf{k}^{\prime} C_{j}(t) \mathbf{k}\right]
\end{aligned}
$$

we are able to derive an evolution equation for the matrix $C_{j}(t)$, namely

$$
\begin{aligned}
\dot{C}_{j}= & 2 \nu E+\Gamma_{j}\left[S_{j j} C_{j}+C_{j} S_{j j}^{T}\right] \\
& +\sum_{l \neq j} \Gamma_{l}\left[S_{j l}\left(\mathbf{x}_{j}-\mathbf{x}_{l}\right) C_{j}+C_{j} S_{j l}\left(\mathbf{x}_{j}-\mathbf{x}_{l}\right)^{T}\right]
\end{aligned}
$$

Here, we explicitly have introduced the matrix $S_{j l}=$ $\left[\nabla \mathbf{U}_{j l}\left(\mathbf{x}_{j}-\mathbf{x}_{l}\right)\right]$ and have singled out the term with $j=l$. The velocity field is now determined from Eq. (12) up to the first order in $C_{j}+C_{l}$ valid for widely separated vortices

$$
\begin{aligned}
\mathbf{U}_{j l}(\mathbf{r}) & =\int \mathrm{d} \mathbf{k}^{\prime} \cdot \mathbf{u}\left(\mathbf{k}^{\prime}\right) e^{-i \mathbf{k}^{\prime} \cdot \mathbf{r}} e^{-\frac{1}{2} \mathbf{k}^{\prime}\left(C_{j}+C_{l}\right) \mathbf{k}^{\prime}} \\
& \approx \mathbf{e}_{z} \times\left[1+\frac{1}{2} \nabla_{\mathbf{r}}\left(C_{j}+C_{l}\right) \nabla_{\mathbf{r}}\right] \frac{\mathbf{r}}{2 \pi|\mathbf{r}|^{2}}
\end{aligned}
$$

The evolution equation for the vortex centers then reads

$$
\begin{aligned}
\dot{\mathbf{x}}_{j}= & \sum_{l} \Gamma_{l} \mathbf{e}_{z} \times \frac{\mathbf{x}_{j}-\mathbf{x}_{l}}{2 \pi\left|\mathbf{x}_{j}-\mathbf{x}_{l}\right|^{2}} \\
& +\sum_{l} \Gamma_{l} \nabla_{\mathbf{x}_{j}}\left[C_{j}+C_{l}\right] \nabla_{\mathbf{x}_{j}} \mathbf{e}_{z} \times \frac{\mathbf{x}_{j}-\mathbf{x}_{l}}{4 \pi\left|\mathbf{x}_{j}-\mathbf{x}_{l}\right|^{2}}
\end{aligned}
$$

A similar system of equations (19) and (21) has been obtained by Melander et al. [25] by means of a truncation of the stream function within their second-order moment model for the Euler equations.

\section{MOTION OF VORTICES WITH EQUAL CIRCULATION WITHIN THE DIFFERENT MODELS}

It is illustrative to consider the interaction of two vortices 1 and 2 at the positions $\mathbf{x}_{1}$ and $\mathbf{x}_{2}$ that possess equal circulation $\Gamma_{1}=\Gamma_{2}=\Gamma$ in the realm of the different vortex models considered above, namely the point vortex model, the Gaussian shape model, and the elliptical Gaussian shape model.

\section{i.) Gaussian shapes:}

Let us consider the case of Gaussian shapes $C_{1}=c_{1} E$ and $C_{2}=c_{2} E$. The symmetry of the problem imposes that $c_{1}=c_{2}=c$, and we arrive at the following evolution equations for the centers

$$
\begin{aligned}
& \dot{\mathbf{x}}_{1}=\Gamma \int \mathrm{d} \mathbf{k}^{\prime} \mathbf{u}\left(\mathbf{k}^{\prime}\right) e^{-i \mathbf{k}^{\prime} \cdot\left(\mathbf{x}_{1}-\mathbf{x}_{2}\right)} e^{-\frac{1}{2} c k^{\prime 2}} \\
& \dot{\mathbf{x}}_{2}=\Gamma \int \mathrm{d} \mathbf{k}^{\prime} \mathbf{u}\left(\mathbf{k}^{\prime}\right) e^{-i \mathbf{k}^{\prime} \cdot\left(\mathbf{x}_{2}-\mathbf{x}_{1}\right)} e^{-\frac{1}{2} c k^{\prime 2}}
\end{aligned}
$$

In making use of

$$
\begin{aligned}
& \frac{i}{4 \pi^{2}} \mathbf{e}_{z} \times \int \mathrm{d} \mathbf{k}^{\prime} \frac{\mathbf{k}^{\prime}}{k^{\prime 2}} e^{-i \mathbf{k}^{\prime} \cdot \mathbf{r}} e^{-\frac{1}{2} c k^{\prime 2}} \\
= & -\frac{1}{4 \pi^{2}} \mathbf{e}_{z} \times \nabla_{\mathbf{r}}\left(\nabla_{\mathbf{r}}^{2}\right)^{-1} \int \mathrm{d} \mathbf{k}^{\prime} e^{-i \mathbf{k}^{\prime} \cdot \mathbf{r}} e^{-\frac{1}{2} c k^{\prime 2}} \\
= & -\frac{1}{2 \pi} \mathbf{e}_{z} \times \nabla_{\mathbf{r}}\left(\nabla_{\mathbf{r}}^{2}\right)^{-1} \frac{\Gamma}{2 \pi c} e^{-\frac{r^{2}}{2 c}} \\
= & \frac{\Gamma}{2 \pi}\left(1-e^{-\frac{r^{2}}{2 c}}\right) \mathbf{e}_{z} \times \frac{\mathbf{r}}{r^{2}}
\end{aligned}
$$


which is the velocity profile of a Lamb-Oseen vortex, the evolution equation for the relative coordinate reads

$$
\dot{\mathbf{r}}=\frac{\Gamma}{\pi}\left(1-e^{-\frac{r^{2}}{2 c}}\right) \mathbf{e}_{z} \times \frac{\mathbf{r}}{r^{2}}
$$

The evolution equation for the shapes $c$ has to be evaluated in a similar fashion from Eq. (19), but for now we invoke the approximation

$$
\dot{c}=2 \nu
$$

where we have neglected the interaction-terms in (19. This yields the evolution equations for two Lamb-Oseen vortices

$$
\begin{aligned}
\dot{r} & =0 \\
\dot{\varphi} & =\frac{\Gamma}{\pi r^{2}}\left(1-e^{-\frac{r^{2}}{4 \nu t}}\right)
\end{aligned}
$$

In the case of vanishing viscosity, we recover the evolution equations of a point vortex pair which undergoes a circular motion around the center $\mathbf{R}=\frac{\mathbf{x}_{1}+\mathbf{x}_{2}}{2}$ with the angular velocity $\frac{\Gamma}{\pi r^{2}}$. Compared to that case, the angular velocity of the Gaussian vortex patches described by Eq. (27) is thus slowed down by viscosity.

However, if we observe such two vortices in real flows, we would see a deformation of the two vortices due to their mutual strain. This deformation in turn, leads to an attractive motion of the vortex centers and ultimately to a merging process of the two vortices. At this point, it is important to notice that a direct consequence of an axisymmetric vorticity profile is that $\dot{r}=0$, which means that no relative motion is induced. Furthermore, in this context we want to mention that a recent investigation of the two-point vorticity statistic in two-dimensional turbulence within a Gaussian approximation revealed the absence of an energy flux from smaller scales to larger scales [24]. The emergence of deformable structures that induce such relative motions in the context of vortex thinning can thus be considered as an important feature of the inverse cascade.

\section{ii.) Elliptical shapes:}

As we have discussed in i.), the mutual interaction of Gaussian vortices in real flows leads to deformations and subsequently attractive motions of the vortex centers. Such deformations can be considered in a first approximation as elliptical deformations.

Therefore, the interaction of two elliptical vortices should for the first time lead to non-vanishing relative motions. The evolution equation for two elliptically shaped vortices read

$$
\begin{aligned}
& \dot{\mathbf{x}}_{1}=\Gamma \int \mathrm{d} \mathbf{k}^{\prime} \mathbf{u}\left(\mathbf{k}^{\prime}\right) e^{-i \mathbf{k}^{\prime} \cdot\left(\mathbf{x}_{1}-\mathbf{x}_{2}\right)} e^{-\frac{1}{2} \mathbf{k}^{\prime}\left[C_{1}+C_{2}\right] \mathbf{k}^{\prime}} \\
& \dot{\mathbf{x}}_{2}=\Gamma \int \mathrm{d} \mathbf{k}^{\prime} \mathbf{u}\left(\mathbf{k}^{\prime}\right) e^{-i \mathbf{k}^{\prime} \cdot\left(\mathbf{x}_{2}-\mathbf{x}_{1}\right)} e^{-\frac{1}{2} \mathbf{k}^{\prime}\left[C_{1}+C_{2}\right] \mathbf{k}^{\prime}}
\end{aligned}
$$

For widely separated vortices the evolution equation for the relative coordinate thus reads

$$
\dot{\mathbf{r}}=\frac{\Gamma}{\pi}\left(1+\frac{1}{2} \nabla_{\mathbf{r}}\left[C_{1}+C_{2}\right] \nabla_{\mathbf{r}}\right) \mathbf{e}_{z} \times \frac{\mathbf{r}}{r^{2}}
$$

which can lead to contributions to the relative motion $\dot{r} \neq 0$, provided that the matrices $C_{1}$ and $C_{2}$ do not reduce to diagonal matrices as in the case of Gaussian shapes. Whether the motion is attractive or repulsive, is to a far extend determined by the alignment angle $\varphi_{a}-\varphi_{r}$ between $\mathbf{r}$ and the major semi axis a of the vortices, which is explicitly derived for the interaction of two rotors in section VIII, for instance in Eq. (45).

\section{THE FORCING MECHANISM}

As it can be seen from Eq. (13), the viscous contributions causes the broadening of the shape of a vortex. Since this effect is more pronounced for smaller vortex structures, thus larger values of $k^{2}$ in (19), an appropriate forcing mechanism has to counteract this effect and provide an energy input at small scales. The forcing mechanism we want to introduce, consists in forcing the semi axes of each elliptical vortex and thus the whole shape of this vortex back to a fixed shape $C_{0}$. It will be seen in section VI that the influence of this kind of forcing makes the two like-signed point vortices of our rotor model to behave as if they were connected by an over-damped spring.

The described forcing mechanism can now be introduced in the following way:

$$
\begin{aligned}
\dot{\Gamma}_{i} & =-a \Gamma_{i}+f_{i} \\
\dot{\mathbf{x}}_{i} & =\sum_{l} \Gamma_{l} \mathbf{U}_{i l}\left(\mathbf{x}_{i}-\mathbf{x}_{l}\right)+\mathbf{U}_{i}(t) \\
\dot{C}_{i} & =2 \nu E+\gamma\left(C_{0}-C_{i}\right)+\Gamma_{i}\left[S_{i i} C_{i}+C_{i} S_{i i}^{T}\right] \\
& +\sum_{l} \Gamma_{l}\left[S_{i l}\left(\mathbf{x}_{i}-\mathbf{x}_{l}\right) C_{i}+C_{i} S_{i l}\left(\mathbf{x}_{i}-\mathbf{x}_{l}\right)^{T}\right]
\end{aligned}
$$

Such type of forcing may be obtained from the vorticity equation (4) by just adding a linear damping term, $-a \omega(\mathbf{k}, t)$ as well as the forcing term $F(\mathbf{k}, t)$,

$$
\begin{aligned}
F(\mathbf{k}, t) & =\sum_{j} f_{j} e^{i \mathbf{k} \cdot \tilde{\mathbf{x}}_{j}(t)+\tilde{W}_{j}} \approx \sum_{j} e^{i \mathbf{k} \cdot \mathbf{x}_{j}(t)+W_{j}} \\
& \times\left[f_{j}+i \Gamma_{j} \mathbf{k} \cdot \mathbf{U}_{j}(t)-\frac{1}{2} \Gamma_{j} \mathbf{k} \gamma\left(C_{0}(t)-C_{j}(t)\right) \mathbf{k}\right]
\end{aligned}
$$

where the centers $\tilde{\mathbf{x}}_{j}=\mathbf{x}_{j}(t)+\frac{\Gamma_{j}}{f_{j}} \mathbf{U}_{j}(t)$ as well as the shapes $\tilde{W}_{j}=W_{j}-\frac{\gamma}{2} \frac{\Gamma_{j}}{f_{j}} \mathbf{k}\left(C_{0}(t)-C_{j}(t)\right) \mathbf{k}$ are close to the centers and the shapes of the elliptical vortices.

The first contribution in Eq. (33) leads to a modulation of the circulation, the second term describes a shift of the rotor center and the third one corresponds to a modification of the width of the Gaussian vortex shape that forces the elliptical vortex back to a certain shape 
$C_{0}$. The stretching of the semi axes of the elliptical vortex due to viscous broadening represented by the first term on the right-hand side in Eq. 32 is thus counteracted by the second term trying to contract the shape of the vortex back to $C_{0}$.

A striking analogy to this forcing mechanism can be found in the explanation of the magneto-rotational instability [26]. Thereby, two elements of an electrically conduct- ing fluid that undergo a rotation around a fixed center are supposed to be connected by an elastic spring repre- senting the magnetic field. As a consequence the angular momentum of the system is not a conserved quantity anymore and the fluid motion becomes unstable.

Although, the introduced forcing mechanism is an adhoc forcing, it emerges in a physically plausible way from the basic equations of the elliptical model 19 and 21. Furthermore, it should be mentioned that the system of equations (30) can be obtained from the Instanton equations of two-dimensional turbulence by means of a variational ansatz with Gaussian elliptical vortices [27].

\section{FORMULATION OF THE ROTOR MODEL}

As we have seen in section IV about the interaction between two point vortices with equal circulation compared to the interaction between two elliptical vortices with equal circulation, the former model fails to describe a relative motion $\dot{r}$ in the direction of $\mathbf{r}$. The thinning mechanism mentioned in 20] is thus clearly neither captured by Onsager's point vortex model nor by a Gaussian distribution of the vorticity, in analogy to [24].

Our vortex model is based on the observation that the point vortex couple considered in section IV] under i.) generates a far field that is similar to that of one elliptical vortex with circulation $\Gamma$. We therefore consider point vortex couples with equal circulation $\Gamma_{i}$ at the positions $\mathbf{x}_{i}$ and $\mathbf{y}_{i}$ as indicated in Fig. 11. The center of this object that we want to term a rotor is then given by $\mathbf{R}_{i}=\frac{\mathbf{x}_{i}+\mathbf{y}_{i}}{2}$. In order to model a forcing and viscous damping mechanism similar to that mentioned in section $\mathrm{V}$ the two point vortices in a rotor are supposed to be glued together by an inelastic spring, such that each rotor possesses an additional degree of freedom and that the size of a single rotor relaxes with relaxation time $1 / \gamma$ to $D_{0}$. Our model then reads

$$
\begin{aligned}
\dot{\mathbf{x}}_{i} & =\frac{\gamma}{2}\left(D_{0}-\left|\mathbf{x}_{i}-\mathbf{y}_{i}\right|\right) \mathbf{e}_{i}+\Gamma_{i} \mathbf{u}\left(\mathbf{x}_{i}-\mathbf{y}_{i}\right) \\
& +\sum_{j} \Gamma_{j}\left[\mathbf{u}\left(\mathbf{x}_{i}-\mathbf{x}_{j}\right)+\mathbf{u}\left(\mathbf{x}_{i}-\mathbf{y}_{j}\right)\right] \\
\dot{\mathbf{y}}_{i} & =-\frac{\gamma}{2}\left(D_{0}-\left|\mathbf{y}_{i}-\mathbf{x}_{i}\right|\right) \mathbf{e}_{i}+\Gamma_{i} \mathbf{u}\left(\mathbf{y}_{i}-\mathbf{x}_{i}\right) \\
& +\sum_{j} \Gamma_{j}\left[\mathbf{u}\left(\mathbf{y}_{i}-\mathbf{y}_{j}\right)+\mathbf{u}\left(\mathbf{y}_{i}-\mathbf{x}_{j}\right)\right]
\end{aligned}
$$

where we have defined the unit vector $\mathbf{e}_{i}=\frac{\mathbf{x}_{i}-\mathbf{y}_{i}}{\left|\mathbf{x}_{i}-\mathbf{y}_{i}\right|}$ and the velocity field $\mathbf{u}(\mathbf{r})$ is the velocity field of a point vortex
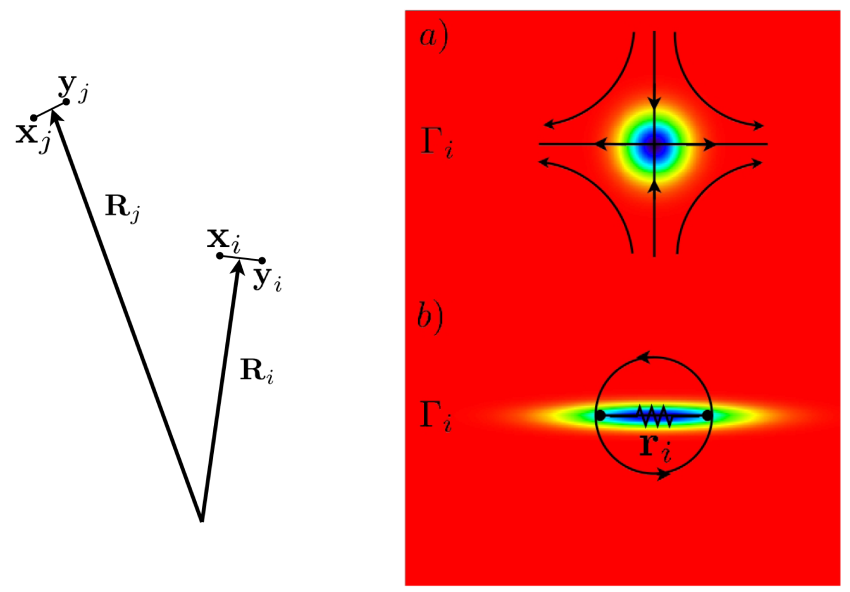

Figure 1. (Color online) left: Interaction of two rotors $\mathbf{R}_{i}$ and $\mathbf{R}_{j}$ with circulation $\Gamma_{i}$ and $\Gamma_{j}$. A rotor is composed of two vortices $\mathbf{x}_{i}$ and $\mathbf{y}_{i}$ with equal circulation $\Gamma_{i}$. For the rotor centered at $\mathbf{R}_{j}$, the circular motion of $\mathbf{x}_{i}$ and $\mathbf{y}_{i}$ around their center coordinate $\mathbf{R}_{i}$ mimics an infitely-thin elliptical vortex with semi axes $\mathbf{r}_{i}=\mathbf{x}_{i}-\mathbf{y}_{i}$, which can be seen from the similarity between the multipole expansion (36) and Eq. (21). This leads to a relative motion between the two rotors $\dot{R}_{i j}=\frac{\mathrm{d}}{\mathrm{d} t}\left|\mathbf{R}_{i}-\mathbf{R}_{j}\right|$, which is not present in the point vortex model and thus can be considered as an important fingerprint of the inverse cascade of two-dimensional turbulence.

right: a) The thinning mechanism of a circular vortex with circulation $\Gamma_{i}$ in a shear velocity field. The rotor in b) is sensitive to such kind of shearing due to his special elliptical shape. It is sheared in the direction of the semi axes $\mathbf{r}_{i}$ and since the elongated structure is exposed to dissipation the vortex tends to broaden away. The forcing mechanism now forces the vortex back to a certain structure, acting as an overdamped spring between the two like-signed vortices of one rotor.

centered at the origin, $\mathbf{u}(\mathbf{r})=\mathbf{e}_{z} \times \frac{\mathbf{r}}{2 \pi r^{2}}$. The first two terms on the right-hand side of equation (34) describe the interaction within one rotor, whereas the last two terms describe the interaction with the other rotors. For vortices moving inside a closed regime, the velocity field has to be changed based on the introduction of mirror vortices [11, 12].

It is important to stress that the above system is not a Hamiltonian system anymore due to the inelastic coupling which mimics an energy input to the system on a scale $D_{0}$. Furthermore, by the additional degree of freedom the rotor is sensitive with respect to a shear velocity field which can be seen from the multipole expansion of the relative coordinate $\mathbf{r}_{i}=\mathbf{x}_{i}-\mathbf{y}_{i}$ with respect to the leading terms in $|\mathbf{r}| /|\mathbf{R}|$, derived in the appendix $\mathrm{A}$

$$
\dot{\mathbf{r}}_{i}=\gamma\left(D_{0}-r_{i}\right) \frac{\mathbf{r}_{i}}{r_{i}}+2 \Gamma_{i} \mathbf{u}\left(\mathbf{r}_{i}\right)+\sum_{j} \Gamma_{j} \mathbf{r}_{i} \cdot \nabla \mathbf{u}\left(\mathbf{R}_{i j}\right)
$$

The influence of the forcing can be seen from the first term: If a rotor is subjected to shear, the spring between 


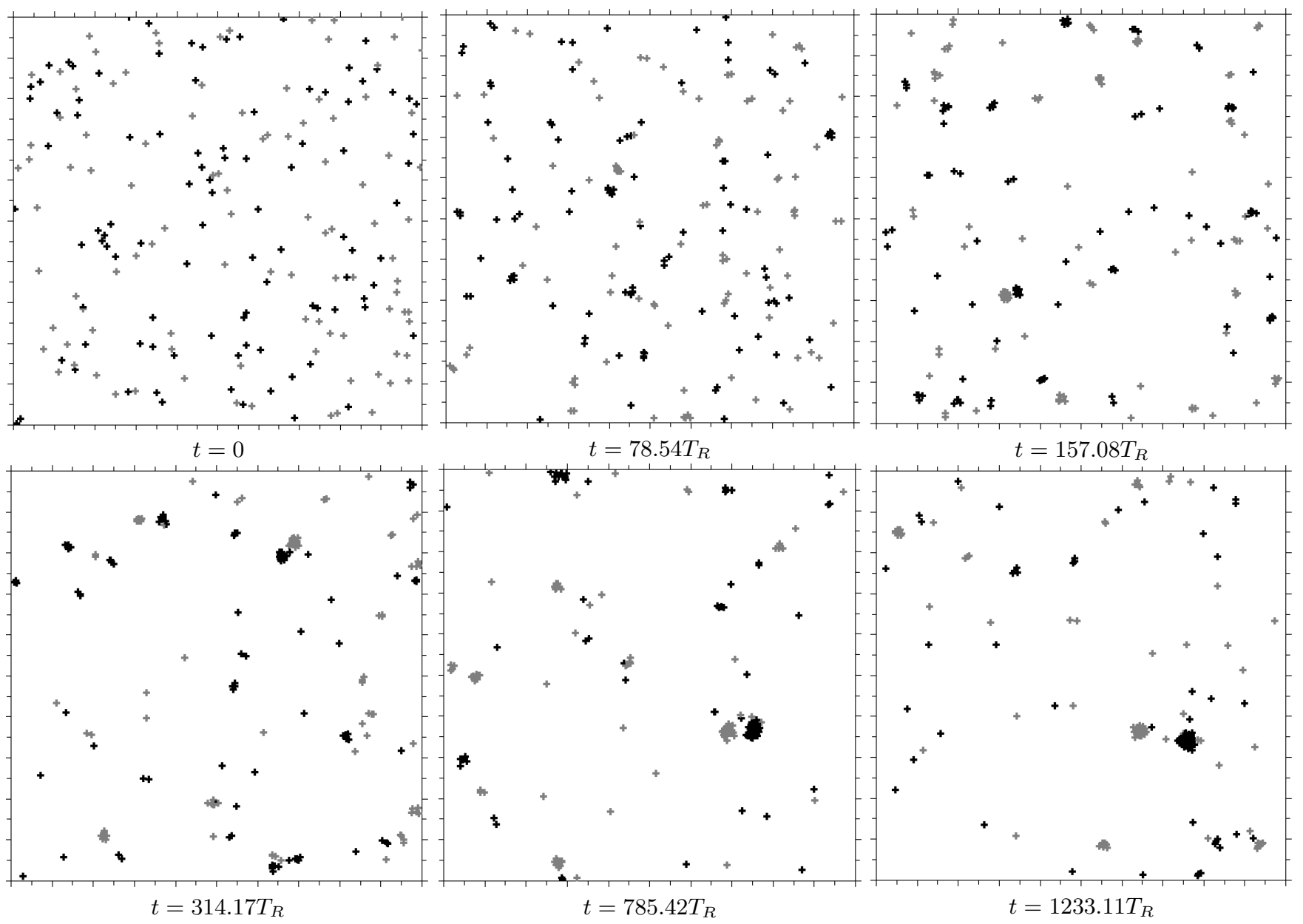

Figure 2. Inverse cascade within the rotor model. Starting from 200 randomly distributed rotors with circulations $\Gamma= \pm 2 \pi$, rotor clusters of like-signed circulation begin to form. Eventually, a dipole-cluster is formed that moves through the box attracting the remaining vortices.

the point vortices in a rotor pulls back and the rotor relaxes to the size $D_{0}$. The shear velocity in the last term is thereby generated by the other rotors.

In a similar way, the multipole expansion of the center coordinate of the rotor in appendix Aleads to the evolution equation

$\dot{\mathbf{R}}_{i}=2 \sum_{j} \Gamma_{j} \mathbf{u}\left(\mathbf{R}_{i j}\right)+\frac{1}{4} \sum_{j} \Gamma_{j}\left[\left(\mathbf{r}_{i} \cdot \nabla\right)^{2}+\left(\mathbf{r}_{j} \cdot \nabla\right)^{2}\right] \mathbf{u}\left(\mathbf{R}_{i j}\right)$

The evolution equation is identical to equation (21), provided that the matrix $C_{i}$ can be written as $C_{i}(t)=$ $\mathbf{r}_{i} \mathbf{r}_{i}$, which corresponds to an infinitely thin elliptical vortex oriented in $\mathbf{r}_{i}$-direction. The relative distance $\mathbf{r}_{i}$ can thus be considered as an elliptical deformation of the velocity field that depends on the shear velocity field induced by the remaining vortices and the effect of the overdamped spring. Furthermore, we again want to emphasize that the last term in Eq. (36) induces relative motions between the rotors as we have seen in section IV] The usual point vortex dynamics solely represented by the first term on the right hand side of equation (36) is thus extended to a dynamical system that is sensitive to the effect of vortex thinning.

\section{NUMERICAL RESULTS}

We have numerically solved the dynamical system (34) in a square periodic domain $L=40$. As a consequence of the periodic setting, the velocity kernels in (34) have to be modified according to

$\mathbf{u}\left(\mathbf{x}_{i}-\mathbf{x}_{j}\right)=\mathbf{e}_{z} \times \sum_{m, n=-N}^{N} \frac{\mathbf{x}_{i}-\mathbf{x}_{j}-m L \mathbf{e}_{x}-n L \mathbf{e}_{y}}{2 \pi\left|\mathbf{x}_{i}-\mathbf{x}_{j}-m L \mathbf{e}_{x}-n L \mathbf{e}_{y}\right|^{2}}$

where the boxes have been continued periodically, with up to $N=5$ layers of neighboring boxes, which guarantees a sufficient degree of homogeneity. The temporal evolution of 200 rotors with an equal number of positive and negative circulations starting from a random initial condition exhibits the formation of a large scale vortical structure via the formation of rotor-clusters. 


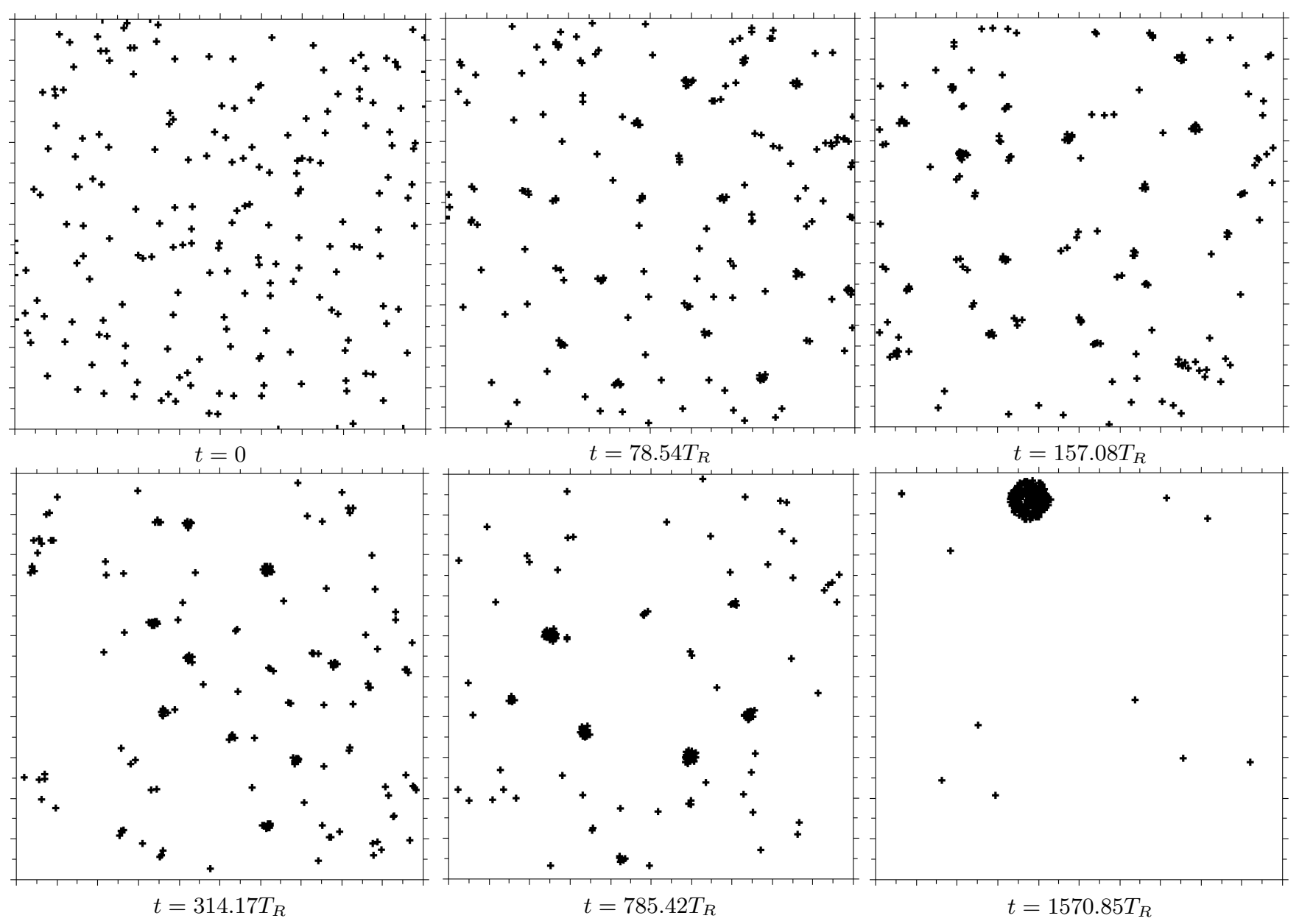

Figure 3. Formation of rotor clusters from 200 randomly distributed vortices with circulation $\Gamma=2 \pi$ in a periodic box $L=40$. The fluctuations of the lattice of rotor clusters ends in a big monopole. The time scale is given in the characteristic rotor turnover time $T_{R}$ of the system.

A typical time series is exhibited in Fig. 2, for the parameter values $\left(N=200, \Gamma= \pm 2 \pi, \gamma=5 ., D_{0}=.5\right.$, $L=40$ ). The temporal evolution of the system can be quantified by the introduction of a characteristic time scale of the system which is given as the period that a rotor possesses at a fixed distance $D_{0}$ and is in the following termed as one rotor turnover time $T_{R}=\frac{\Gamma}{2 \pi^{2} D_{0}^{2}}$, which follows from equation (27) for the case of vanishing viscosity.

As it can be seen from Fig. 2, the clustering of likesigned rotors already occurs within the first 100 rotor turnover times, which means that the separation of the rotors takes place on a relatively short time-scale. The temporal evolution of 200 rotors with identical circulations starting from random initial positions of the rotors, is exhibited in Fig. 3. A fluctuating lattice of rotor clusters appears and after approximately 1500 rotor turnover times, the system forms a monopole which attracts the remaining rotors.

We have calculated the kinetic energy spectra of the rotor system with $\Gamma= \pm 2 \pi$ at different times in Fig. 4 .
Starting from 20 different initial configurations of the rotors, we let the systems evolve in time and performed the ensemble average at a specific time $t$. Thereby, the spectrum is calculated from the velocity field in Eq. (3) that has been interpolated on a grid and then transformed into Fourier space.

Initially, the rotors possess a clear point vortex spectrum following a power law $E(k, t=0) \sim k^{-1}$. Only at high values of $k$ deviations due to the singular structure of the vorticity and corresponding discontinuities in the velocity field manifest themselves in an increase of $E(k, t)$. This effect can be observed in the following spectra, too. However, after a few $(\approx 100)$ rotor turnover times, as the rotor clustering sets in, a more universal energy spectrum can be observed. Due to an energy flux from smaller to larger scales, the spectra begin to steepen for smaller $k$ values, revealing a spectrum that is close to the predicted $E(k, t) \sim k^{-5 / 3}$. As it can be seen from the compensated spectra in Fig. 4, this slope remains constant for nearly $500 T_{R}$ and an energy flux into the large scales takes place. This is also in agreement with the time-averaged spectral energy flux $\Pi(k, t)$, depicted in Fig. 5 . The inlet plot in 

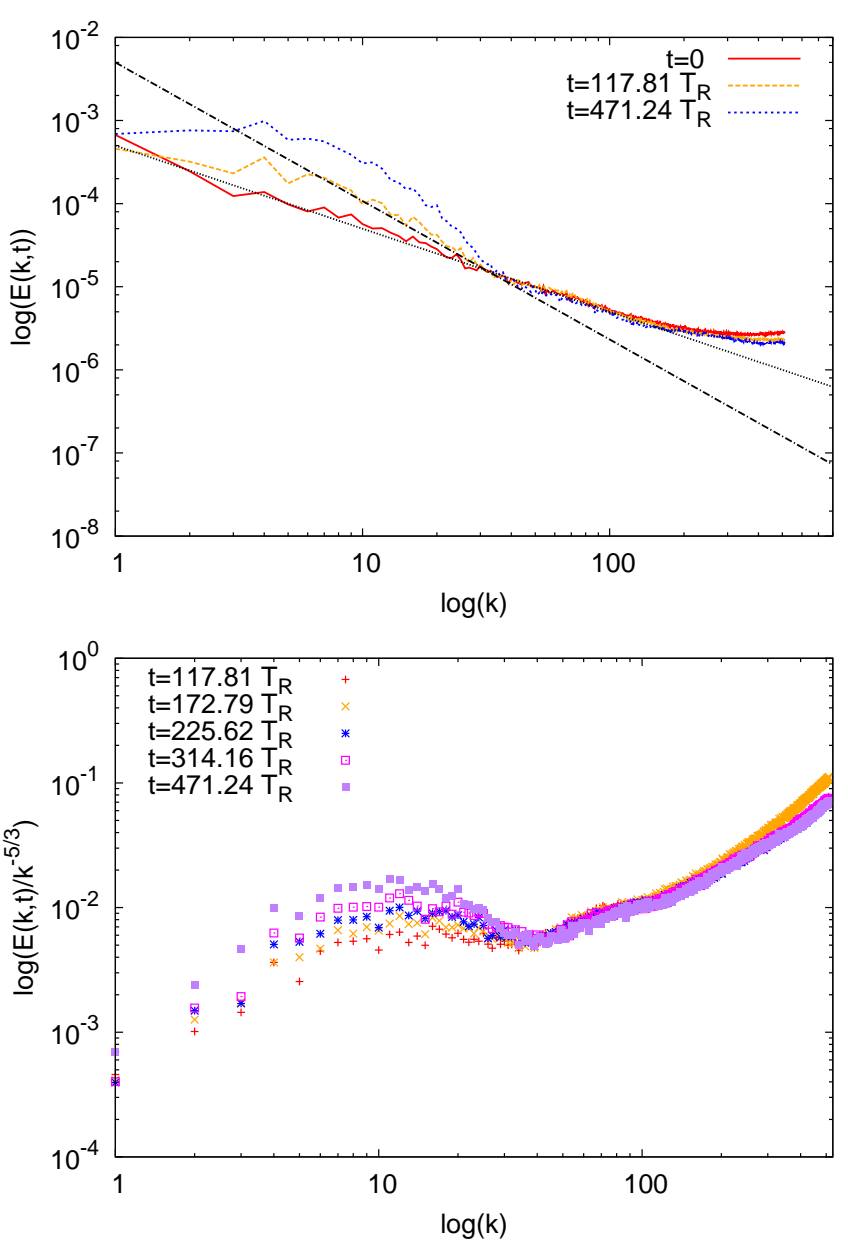

Figure 4. (Color online) Above: Kinetic energy spectra of an assembly of rotors with $\Gamma+ \pm 2 \pi$ as in Fig. 2 for different times $t$. Initially, the spectrum shows the charasteristic of a point vortex spectrum. The dotted line corresponds to $0.0005 k^{-1}$. As time evolves, the spectra begin to steepen corresponding to an energy transfer into larger scales due to the effect of thinning. The dash-dotted line corresponds to $0.005 k^{-5 / 3}$.

Below: Compensated kinetic energy spectra from above. Only after $t \approx 100 T_{R}$, the spectra show the characteristic $5 / 3$-slope, which is in large part maintained over $\approx 300 T_{R}$.

Fig. 5 corresponds to the kinetic energy transfer rate $T(k, t)$, which is related to $\Pi(k, t)$ according to [28]

$$
\Pi(k, t)=\int_{k}^{\infty} \mathrm{d} k^{\prime} T\left(k^{\prime}, t\right)
$$

It is obvious that energy accumulates at small $k$-values. This is not surprising, since the rotor model only provides an energy input on small scales and it will be a task for the future to extend the model in order to achieve a damping at small values of $k$ and thus to extract energy at the integral scale.

We now turn to the determination of the Kolmogorov constant of the energy spectrum from the binary rotor system $(\Gamma= \pm 2 \pi)$. The spectrum as it was predicted by

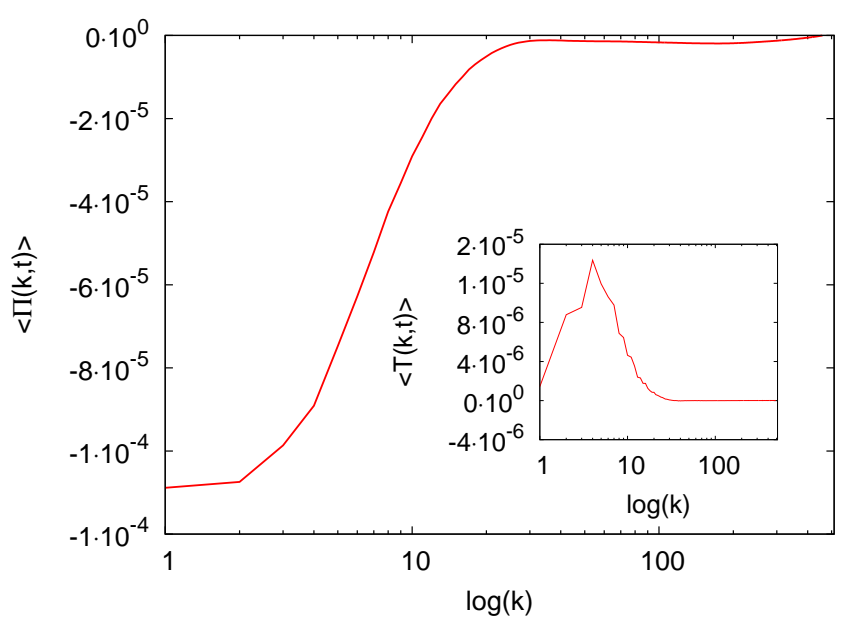

Figure 5. (Color online) Time-averaged kinetic energy flux $\langle\Pi(k, t)\rangle$ calculated from the spectra with 5/3-slope in Fig. 4 An energy transfer into the large scales can clearly be observed. The inlet corresponds to the time-averaged kinetic energy transfer rate $\langle T(k, t)\rangle$.

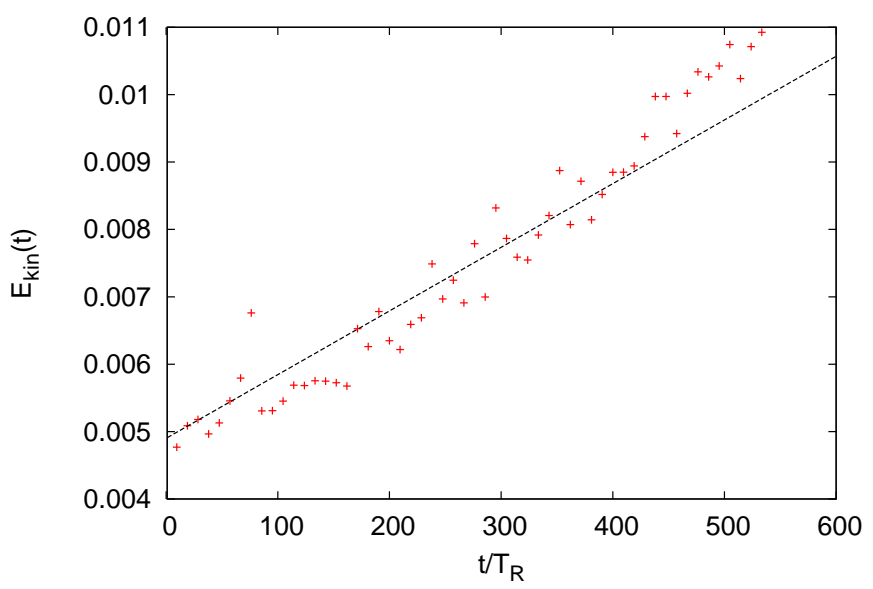

Figure 6. (Color online) Temporal evolution of the kinetic energy of the binary rotor system from Fig. 2, The fitted line possesses a slope $\epsilon=(0.908 \pm 0.052) \cdot 10^{-4}$.

Kraichnan [18] reads

$$
E(k)=C_{K} \epsilon^{2 / 3} k^{-5 / 3}
$$

where $\epsilon$ is the energy dissipation rate.

In the following, $\epsilon$ is determined from the timedependence of the total kinetic energy that shows up to be linear in time within $\approx 5 \%$, proving that the model is not Hamiltonian anymore due to the inclusion of the forcing term. The corresponding plot is depicted in Fig. 6.

The slope of the fitted line can thus be interpreted as the rate of energy input into the system and we obtain a value of $\epsilon=(0.908 \pm 0.052) \cdot 10^{-4}$. In order to make an estimate for $C_{K} \epsilon^{2 / 3}$, we take an average of the compensated 
spectra in Fig. 4 of times between $120 T_{R}$ and $620 T_{R}$ which yields $C_{K} \epsilon^{2 / 3}=0.0119 \pm 0.0015$. The Kolmogorov constant $C_{K}$ of the rotor system for times t between $120 T_{R}$ and $620 T_{R}$ thus lies in the range $C_{K}=5.89 \pm 0.74$. The high inaccuracy of our estimate is due to the estimation of $C_{K} \epsilon^{2 / 3}$. Reported values from direct numerical simulations [29 31] and experiments [32, 33] lie within the range from 5.8 to 7.0 . The Kolmogorov constant of the rotor system thus lies on the lower end of that range. In comparison to the point vortex model of Siggia and Aref [23], who report a Kolmogorov constant of $C_{K}=14$ which is twice the accepted value, the rotor model thus seems to provide an efficient mechanism for the energy transfer upscale due to the effect of vortex thinning.

Another important way to determine the distribution and the occuring structures in the rotor model will be discussed in the following. In order to quantify the emergence of the rotor clusters in Fig. 2 and 3, we make use of the radial distribution function $g(\mathbf{r})$ which can be considered as the probability of finding a like-signed rotor at a distance $\mathbf{r}$ away from a reference-rotor (for further references see for instance [34]). The radial distribution function is therefore given as

$$
g(\mathbf{r})=\frac{1}{\rho}\left\langle\sum_{i, j}^{\prime} \delta\left(\mathbf{x}_{i}-\mathbf{x}_{j}-\mathbf{r}\right)\right\rangle
$$

where $\rho=\frac{L^{2}}{N^{2}}$ and the prime indicates that summation over $i=j$ is left out. The averaging is performed in such a way that the number of like-signed rotors populating a concentric segment of radius $\mathrm{d} r$ at a given radius $\mathrm{r}$ is divided by its area. In the following the radial distribution function is assumed to be isotropic, so that $g(\mathbf{r})=g(r)$. For a disordered state one expects the radial distribution function to be equal to 1 for every $r$. As the formation of the rotor clusters sets in, one should observe an increase of $g(r)$ for small $r$, since the probability of finding a like-signed rotor in the neighborhood of a referencerotor increases.

The radial distribution functions for the two time series are plotted in Fig. 7 and one clearly observes an increase of $g(r)$ at small $r$. In order to get smooth curves, $g(r)$ was calculated in such a way that it shows no discontinuities for $r \approx 0$ due to a minimum distance between neighboring rotors. The radial distribution function can thus be used as a qualitative measure for the formation of the clusters and their typical sizes. Furthermore, the radial distribution function is related to the structure factor

$$
S(\mathbf{q})=1+\frac{1}{N}\left\langle\sum_{i, j}^{\prime} e^{-i \mathbf{q} \cdot\left(\mathbf{x}_{i}-\mathbf{x}_{j}\right)}\right\rangle
$$

in a way that

$$
\begin{aligned}
S(\mathbf{q}) & =1+\rho \int \mathrm{d} \mathbf{r}(g(r)-1) e^{-i \mathbf{q} \cdot \mathbf{r}} \\
& =1+2 \pi \rho \int_{0}^{\infty} \mathrm{d} r r(g(r)-1) J_{0}(q r)
\end{aligned}
$$
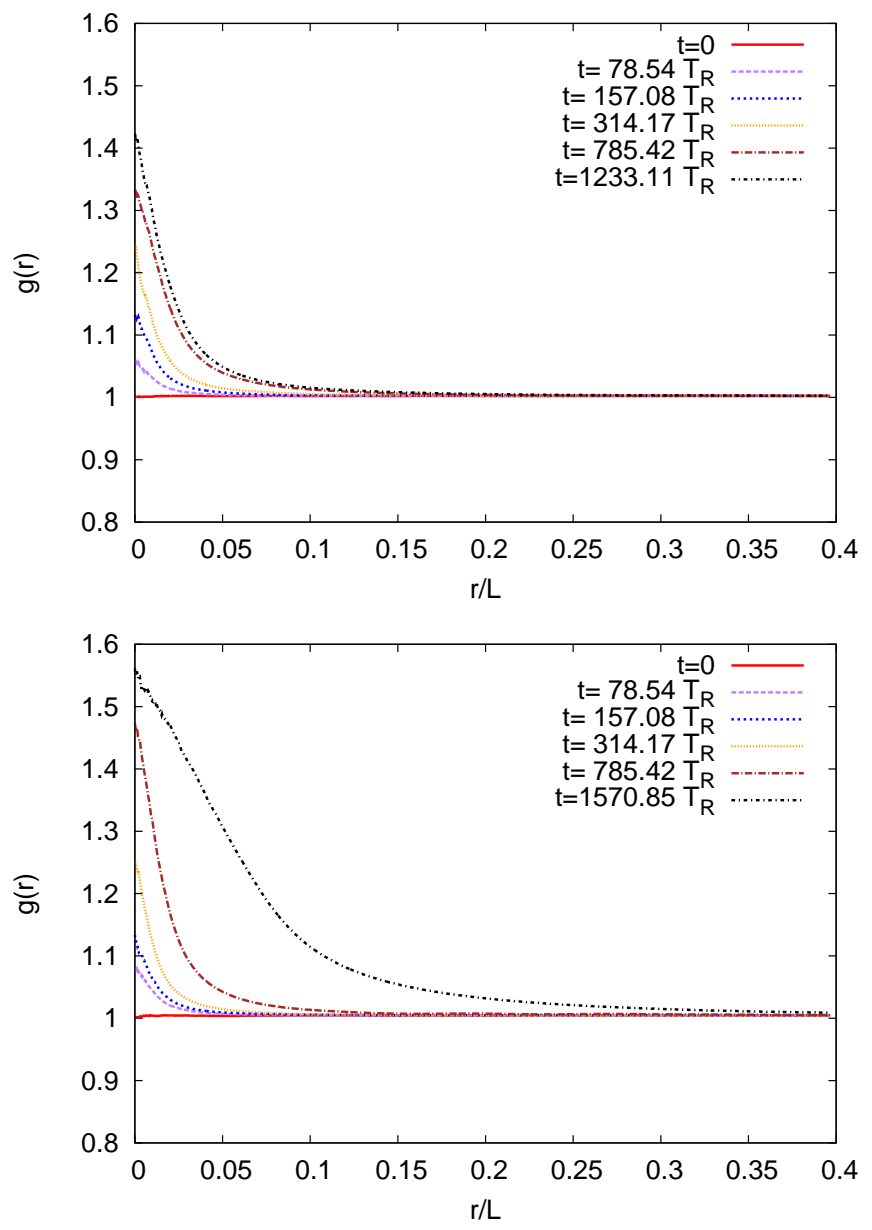

Figure 7. (Color online) Above: Radial distribution functions for six different times from the time series of rotors with $\Gamma=$ $\pm 2 \pi$ in Fig. 2. An increase of $g(r)$ for small $r$ can be observed meaning an increased probability of finding a like-signed rotor in the neighborhood of a reference-rotor.

Below: Radial distribution functions for six different times from the time series of the rotors with $\Gamma=2 \pi$ in Fig. 3 Again, an increase of $g(r)$ can be observed. The formation of the final monopole manifests itself in a long-ranging $g(r)$.

where $J_{0}(q r)$ is the Bessel function of order zero. The structure factor $S(q)$ can thus be calculated via the Hankel transform of $(g(r)-1)$, provided that the radial distribution function is isotropic. The structure factors for the two system are plotted in Fig. 8, For the case of the mixed system of Fig. 2, one observes an increase of $S(q)$ over time. Whether this increase is governed by a power law for intermediate $q$ has to be evaluated within further simulations of the model equations (34). Furthermore, Eq. (42) is of great importance for the investigation of the rotor model, since it relates macroscopic quantities on the left-hand side to microscopic quantites such as the radial distribution function. It is thus a good starting point for the interpretation of the fluctuations of the rotor clusters in the realm of phase transitions.

The growth rate of the rotor clusters can be determined 

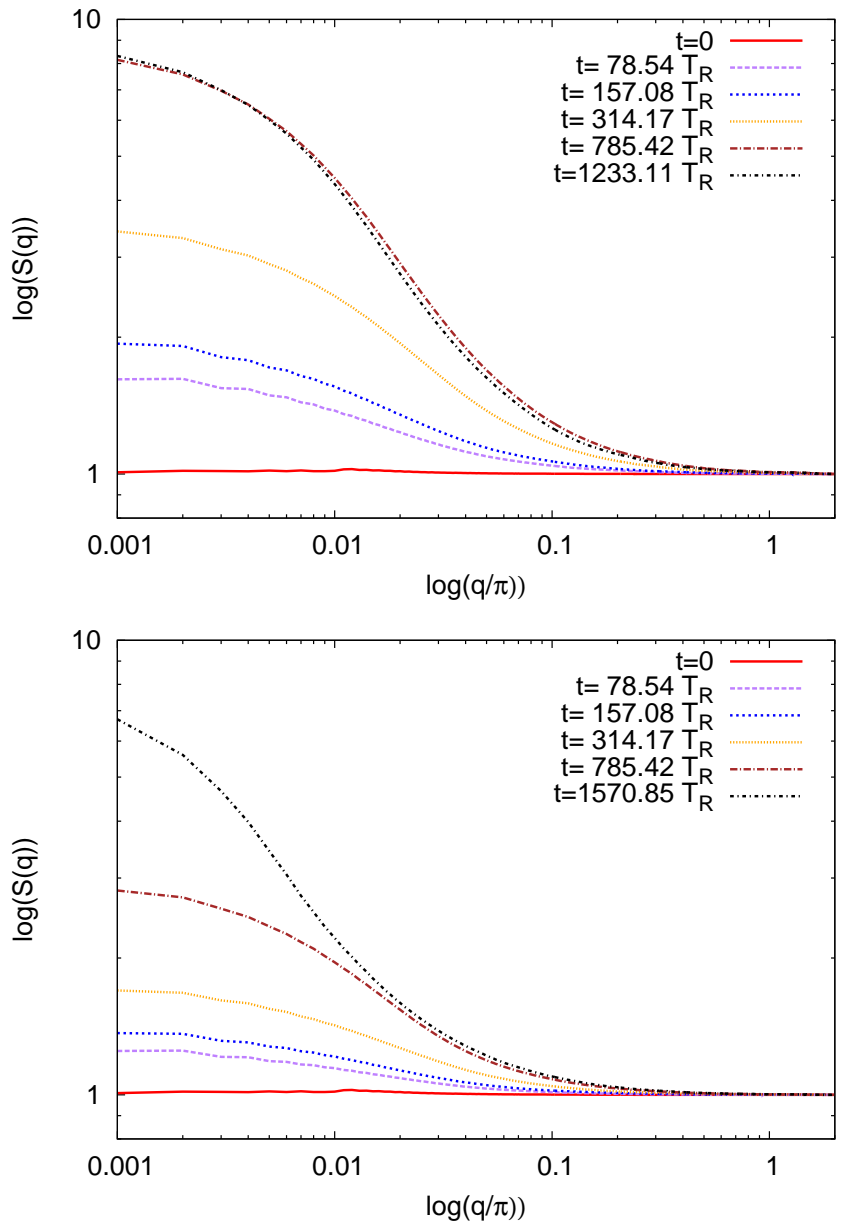

Figure 8. (Color online) Above: Structure factors $S(q)$ from the radial distribution function of the time series of rotors with $\Gamma= \pm 2 \pi$ in Fig. 2 ,

Below: Structure factors $S(q)$ from the radial distribution function of the time series of rotors with $\Gamma=2 \pi$ in Fig. 3 The flucuations of the rotor lattice is accompagnied by an increase of the structure factor $S(q)$ over time.

from the time dependence of the structure factor. The growth of the largest structures of the system is given by $S(q=0, t)$. In Fig. 9, the temporal evolution of $S(q=0, t)$ is plotted for the two systems. The fluctuating rotor lattice below exhibits a pronounced growth rate after $t \approx 600 T_{R}$, whereas the growth rate of the mixed system above already increases for $t \approx 150 T_{R}$. For comparison, two power laws $t^{0.2}$ and $t^{1.3}$ were plotted in the figures. The growth rate of our rotor clusters can thus be considered as relatively strong compared to typical growth rates from pattern formation, for instance compared to the growth rate of droplets in the Cahn-Hilliard equation where $S(q=0, t) \sim t^{1 / 3}$ according to SlyozovLifshitz theory [35].

The fact that the rotor vortex system exhibits a pronounced inverse cascade already for moderate numbers of rotors (200 rotors have been used for the figures) on a small time-scale allows us to investigate the inverse cas-
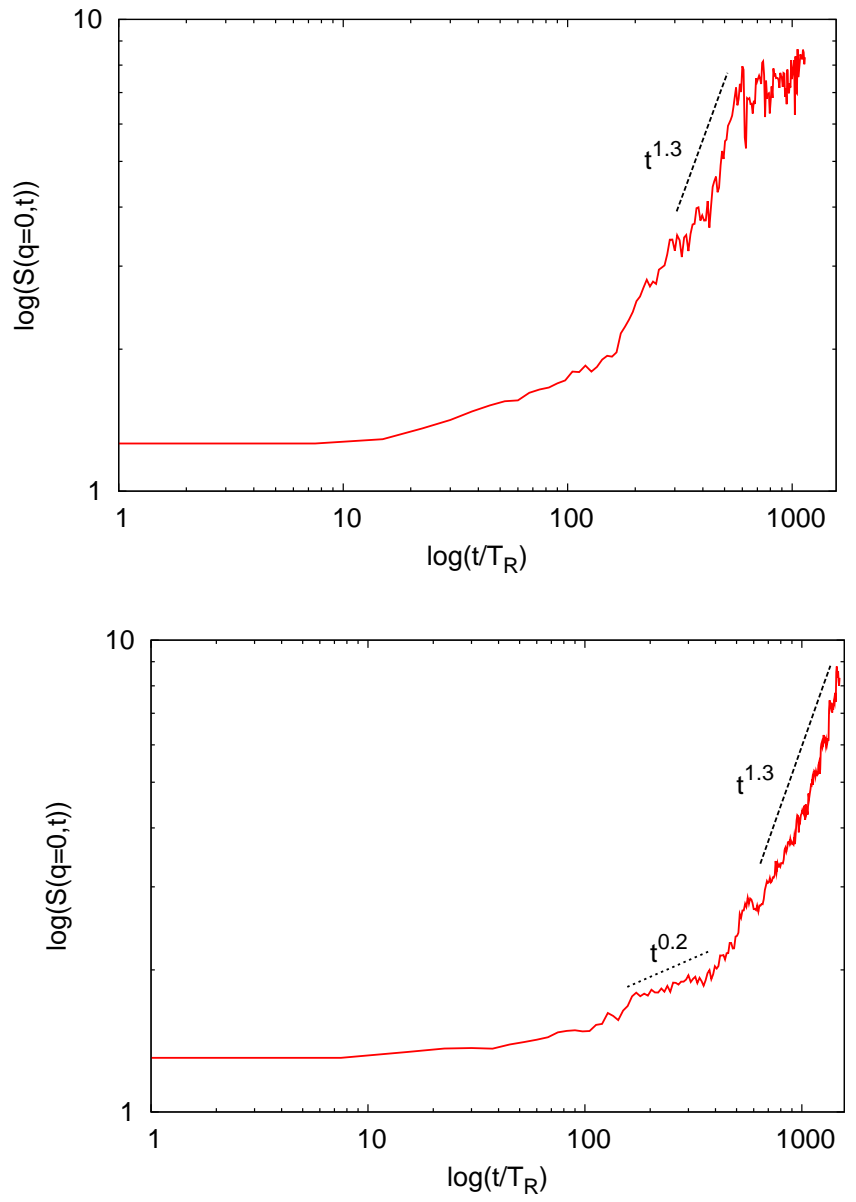

Figure 9. (Color online) Above: Temporal evolution of $S(q=$ $0, t)$ of the time series of rotors with $\Gamma= \pm 2 \pi$ in Fig. 2. A power law with $t^{1.3}$ is plotted for comparison. The strong growth rate saturates after $t \approx 500 T_{R}$.

Below: Temporal evolution of $S(q=0, t)$ of the time series of rotors with $\Gamma=2 \pi$ in Fig. 3. Two power laws $t^{0.2}$ and $t^{1.3}$ are plotted for comparison.

cade using methods of nonlinear dynamics. Although, usual point vortex models such as [23], have been known for a long time to possess inverse energy cascades the present model incorporates the aspect of vortex thinning, due to a possible change of the ellipticity of the rotor in much the same way as identified in the experiments of Chen et al 20]. Hence, it is a minimal dynamical model containing the mechanisms of the inverse cascade. In the following we shall discuss the origin of the formation of clusters of rotors with like-signed circulations.

\section{INTERACTION OF TWO ROTORS}

As it has been discussed in section IV the deformation of the shapes of the vortices induces relative motion between their centers. However, the analytical calculation of these relative velocities directly from the fluid dynami- 
cal equations as it has been performed for instance in [25], are quite difficult to handle in order to get meaningful results of the dynamics underlying the inverse cascade. However, since our model possesses two kinds of dynamics, i.e. a fast dynamic within the rotation of the point vortex pairs in one rotor and a slower dynamic within the interaction between the rotors, it is possible to simplify the corresponding equations within an adiabatic approximation of the fast rotations. The result for the relative motion between two rotors reveals the importance for the dynamical aspects of the inverse cascade caused by an attractive motion in between two like-signed vortices and the symmetry-breaking $\Gamma_{i} \rightarrow-\Gamma_{i}$ due to the introduction of the forcing in Eq. (34).

In the following, we consider the configuration of two rotors with circulations $\Gamma_{i}$ and $\Gamma_{j}$, depicted in Fig. 1. which can be considered as the interaction of two infinitely-thin elliptical vortices in the same manner as ii.) from section [V] It is straightforward to show that the center of vorticity $\frac{\left(\Gamma_{i} \mathbf{R}_{i}+\Gamma_{j} \mathbf{R}_{j}\right)}{\Gamma_{i}+\Gamma_{j}}$ is a conserved quantity. The distance vector $\mathbf{R}_{i j}=\mathbf{R}$ between the two rotors obeys the evolution equation

$$
\begin{aligned}
\dot{\mathbf{R}}= & \frac{\left(\Gamma_{i}+\Gamma_{j}\right)}{\pi} \mathbf{e}_{z} \times\left[\frac{\mathbf{R}}{|\mathbf{R}|^{2}}\right. \\
& +\frac{1}{8}\left[-2 \frac{\mathbf{R}}{|\mathbf{R}|^{4}} \mathbf{r}_{i}^{2}-4 \frac{\mathbf{r}_{i}}{|\mathbf{R}|^{4}} \mathbf{r}_{i} \cdot \mathbf{R}+8 \frac{\mathbf{R}}{|\mathbf{R}|^{6}}\left(\mathbf{r}_{i} \cdot \mathbf{R}\right)^{2}\right] \\
& \left.+\frac{1}{8}\left[-2 \frac{\mathbf{R}}{|\mathbf{R}|^{4}} \mathbf{r}_{j}^{2}-4 \frac{\mathbf{r}_{j}}{|\mathbf{R}|^{4}} \mathbf{r}_{j} \cdot \mathbf{R}+8 \frac{\mathbf{R}}{|\mathbf{R}|^{6}}\left(\mathbf{r}_{j} \cdot \mathbf{R}\right)^{2}\right]\right]
\end{aligned}
$$

which follows from equation (36) in calculating the corresponding velocity field gradients, described in the appendix $\mathrm{A}$.

For the following it is convenient to represent the unit vectors according to $\mathbf{e}_{i}=\frac{\mathbf{r}_{i}}{r_{i}}=\left(\begin{array}{c}\cos \varphi_{i} \\ \sin \varphi_{i}\end{array}\right)$, as well as $\mathbf{e}_{R}=\frac{\mathbf{R}}{R}=\left(\begin{array}{c}\cos \varphi_{R} \\ \sin \varphi_{R}\end{array}\right)$, which yields

$$
\mathbf{e}_{R} \cdot\left[\mathbf{e}_{z} \times \mathbf{e}_{i}\right]\left(\mathbf{e}_{i} \cdot \mathbf{e}_{R}\right)=\frac{1}{2} \sin \left(2\left(\varphi_{i}-\varphi_{R}\right)\right)
$$

We obtain the equation for the relative distance

$$
\begin{aligned}
\dot{R}=-\frac{\left(\Gamma_{i}+\Gamma_{j}\right)}{4 \pi} \frac{1}{R^{3}} & {\left[r_{i}^{2} \sin \left(2\left(\varphi_{i}-\varphi_{R}\right)\right)\right.} \\
& \left.+r_{j}^{2} \sin \left(2\left(\varphi_{j}-\varphi_{R}\right)\right)\right]
\end{aligned}
$$

The evolution equation for the relative coordinate of a rotor reads

$$
\begin{aligned}
\dot{\mathbf{r}}_{i}= & \gamma\left(D_{0}-r_{i}\right) \frac{\mathbf{r}_{i}}{r_{i}}+\frac{\Gamma_{i}}{\pi} \mathbf{e}_{z} \times \frac{\mathbf{r}_{i}}{r_{i}^{2}} \\
& +\frac{\Gamma_{j}}{2 \pi} \mathbf{e}_{z} \times\left[\frac{\mathbf{r}_{i}}{|\mathbf{R}|^{2}}-2 \frac{\mathbf{R}}{|\mathbf{R}|^{4}}\left(\mathbf{r}_{i} \cdot \mathbf{R}\right)\right]
\end{aligned}
$$

which follows from equation (35) and the calculation of the velocity field gradients, performed in the appendix
A. We have to determine the quantities $r_{i}^{2}, r_{j}^{2}$, which are determined by the evolution equations

$$
\begin{gathered}
\dot{r}_{i}=\gamma\left(D_{0}-r_{i}\right)+\frac{\Gamma_{j}}{2 \pi} \frac{r_{i}}{R^{2}} \sin \left(2\left(\varphi_{i}-\varphi_{R}\right)\right) \\
\dot{r}_{j}=\gamma\left(D_{0}-r_{j}\right)+\frac{\Gamma_{i}}{2 \pi} \frac{r_{j}}{R^{2}} \sin \left(2\left(\varphi_{j}-\varphi_{R}\right)\right)
\end{gathered}
$$

We can solve iteratively for small deviations of $r_{i}$ from $D_{0}$ :

$r_{i}=D_{0}+\frac{\Gamma_{j}}{2 \pi} \frac{D_{0}}{R^{2}} \int_{-\infty}^{t} \mathrm{~d} t^{\prime} e^{-\gamma\left(t-t^{\prime}\right)} \sin \left(2\left(\varphi_{i}\left(t^{\prime}\right)-\varphi_{R}\left(t^{\prime}\right)\right)\right)$

A similar treatment applies to $r_{j}$. Splitting the rotation into its fast $\left(\sim e^{2 i\left(\omega_{i}-\omega_{R}\right) t}\right)$ and slow varying parts $e^{2 i\left(\tilde{\varphi}_{i}(t)-\tilde{\varphi}_{R}(t)\right)}$, i.e.

$$
e^{2 i\left(\varphi_{i}(t)-\varphi_{R}(t)\right)} \approx e^{2 i\left(\omega_{i}-\omega_{R}\right) t} e^{2 i\left(\tilde{\varphi}_{i}(t)-\tilde{\varphi}_{R}(t)\right)}
$$

we obtain after a partial integration

$$
\begin{aligned}
& \int_{-\infty}^{t} \mathrm{~d} t^{\prime} e^{-\gamma\left(t-t^{\prime}\right)} e^{2 i\left(\tilde{\varphi}_{i}\left(t^{\prime}\right)-\tilde{\varphi}_{R}\left(t^{\prime}\right)\right)} \\
& =\frac{e^{2 i\left(\omega_{i}-\omega_{R}\right) t} e^{2 i\left(\tilde{\varphi}_{i}(t)-\tilde{\varphi}_{R}(t)\right)}}{2 i\left(\omega_{i}-\omega_{R}\right)+\gamma} \\
& -\int_{-\infty}^{t} \mathrm{~d} t^{\prime} e^{-\gamma\left(t-t^{\prime}\right)+2 i\left(\omega_{i}-\omega_{R}\right) t^{\prime}}\left[\left(\omega_{i}-\omega_{R}\right)+\frac{\gamma}{2 i}\right]^{-1} \\
& \times e^{2 i\left(\tilde{\varphi}_{i}\left(t^{\prime}\right)-\tilde{\varphi}_{R}\left(t^{\prime}\right)\right)}\left(\dot{\tilde{\varphi}}_{i}\left(t^{\prime}\right)-\dot{\tilde{\varphi}}_{R}\left(t^{\prime}\right)\right)
\end{aligned}
$$

In order to proceed with the adiabatic approximation, we neglect the second term in Eq. (50) since it contains time derivatives of the slowly varying parts of the rotations. Assuming that the damping constant $\gamma$ is large compared to the rotation frequency of the rotor, we obtain

$$
\begin{aligned}
& \int_{-\infty}^{t} \mathrm{~d} t^{\prime} e^{-\gamma\left(t-t^{\prime}\right)} \sin \left(2\left(\varphi_{i}\left(t^{\prime}\right)-\varphi_{R}\left(t^{\prime}\right)\right)\right) \\
& \approx \frac{\sin \left(2\left(\varphi_{i}(t)-\varphi_{R}(t)\right)\right)}{\gamma}
\end{aligned}
$$

To lowest order in $\gamma^{-1}$ we thus obtain

$$
\begin{aligned}
& r_{i}^{2}=D_{0}^{2}\left[1+\frac{\Gamma_{j}}{\pi \gamma R^{2}} \sin \left(2\left(\varphi_{i}-\varphi_{R}\right)\right)\right] \\
& r_{j}^{2}=D_{0}^{2}\left[1+\frac{\Gamma_{i}}{\pi \gamma R^{2}} \sin \left(2\left(\varphi_{j}-\varphi_{R}\right)\right)\right]
\end{aligned}
$$

Here, the last terms on the right-hand side arise due to the change of the size of the rotors, connected with a change of the far field, induced by the mutually generated shear. It thus mimics the mechanism of vortex thinning, identified in [20].

The relative motion of the rotors obeys the evolution equation

$$
\begin{aligned}
\dot{R}= & -\frac{\left(\Gamma_{i}+\Gamma_{j}\right)}{4 \pi} \frac{D_{0}^{2}}{R^{3}}\left[\sin \left(2\left(\varphi_{i}-\varphi_{R}\right)\right)+\sin \left(2\left(\varphi_{j}-\varphi_{R}\right)\right)\right. \\
& \left.+\frac{\Gamma_{j}}{\pi \gamma R^{2}} \sin ^{2}\left(2\left(\varphi_{i}-\varphi_{R}\right)\right)+\frac{\Gamma_{i}}{\pi \gamma R^{2}} \sin ^{2}\left(2\left(\varphi_{j}-\varphi_{R}\right)\right)\right]
\end{aligned}
$$


We now average the evolution equation with respect to the rotations of the vectors $\mathbf{e}_{i}(t)$ and $\mathbf{e}_{j}(t)$ taking into account that the averages $\left\langle\sin \left(2\left(\varphi_{i}-\varphi_{R}\right)\right)\right\rangle=$ $\left\langle\sin \left(2\left(\varphi_{j}-\varphi_{R}\right)\right)\right\rangle=0$ vanish. Furthermore, the averages $\left\langle\sin ^{2}\left(2\left(\varphi_{i}-\varphi_{R}\right)\right)\right\rangle=\left\langle\sin ^{2}\left(2\left(\varphi_{j}-\varphi_{R}\right)\right)\right\rangle=a$ are positive. As a consequence, the relative distance behaves according to

$$
\dot{R}=-\frac{\left(\Gamma_{i}+\Gamma_{j}\right)^{2}}{(2 \pi)^{2}} \frac{D_{0}^{2}}{\gamma R^{5}} a
$$

Two rotors approach each other, except for $\Gamma_{i}=-\Gamma_{j}$. It is important to stress that this attractive relative motion arises only if we include the irreversible effect of the strain induced stretching of the rotors. Furthermore, the symmetry breaking of $\Gamma_{i} \rightarrow-\Gamma_{i}$ in equation (53) can be considered as an important feature of the rotor model in comparison to the point vortex model, which conserves this symmetry.

\section{CONCLUSIONS}

We have presented a generalized point vortex model, a rotor model, exhibiting an inverse cascade based on clustering of rotors. We have discussed how this rotor model can be derived from the vorticity equation by an expansion of the vorticity field into a set of elliptical vortices at locations $\mathbf{x}_{i}(t)$ and shapes $C_{i}(t)$. An important point has been the inclusion of a forcing term, which prevents the elliptical far field of the rotors from diffusing away. The added forcing term breaks the symmetry $\Gamma_{i} \rightarrow-\Gamma_{i}$, $t \rightarrow-t$. This symmetry breaking lies at the origin of cluster formation and the inverse cascade, as can be seen from the two-rotor interaction inducing in average a relative motion proportional to $\frac{D_{0}^{2}}{\gamma}\left(\Gamma_{1}+\Gamma_{2}\right)^{2} / R^{5}$.

The numerical simulations of the model equations (34) reveal the formation of rotor clusters on a short time scale. In addition, the calculated energy spectra and energy fluxes give strong evidence for the important role of vortex thinning during the cascade process in twodimensional turbulence.

The presented rotor model can be investigated by applying methods from dynamical systems theory like the evaluation of finite time Ljapunov exponents and Ljapunov vectors. These and further dynamical aspects are the basis for future work and will be covered in a following paper. The model system (30) may also be studied as a stochastic system by considering the velocity $\mathbf{U}_{i}(t)$ to be a white noise force. The corresponding FokkerPlanck equation allows one to draw analogies with quantum mechanical many body problems. Furthermore, we emphasize that a continuum version of the model equations (30) leads to a subgrid model exhibiting analogies with the work of Eyink [36].

It will be a task for the future to investigate the cluster formation from a statistical point of view, based on the formulation of kinetic equations, along the lines as has been performed for fully developed turbulence 37 39], and Rayleigh-Bénard convection [40]. In this respect we hope to find a relation to the kinetic equation for the two-point vorticity statistics recently derived on the basis of the Monin-Lundgren-Novikov hierarchy, taking conditional averages from direct numerical simulations [24].

\section{ACKNOWLEDGMENTS}

J.F. is very grateful for discussions with Michael Wilczek and Frank Jenko about the organization of this paper. Sadly, Rudolf Friedrich ( $\dagger 16$ th August 2012) unexpectedly passed away during this work. He was as much an inspiring physicist as well as a caring father.

\section{Appendix A}

In this part we calculate the multipole expansion of a rotor, defined by $\mathbf{x}_{i}$ and $\mathbf{y}_{i}$ in Fig. 1. To this end, we introduce relative and center coordinates, according to

$$
\mathbf{r}_{i}=\mathbf{x}_{i}-\mathbf{y}_{i} \quad \text { and } \quad \mathbf{R}_{i}=\frac{\mathbf{x}_{i}+\mathbf{y}_{i}}{2}
$$

as well as the vector $\mathbf{R}_{i j}=\mathbf{R}_{i}-\mathbf{R}_{j}$.

In using equation (34), we obtain the evolution equation for the relative coordinate

$$
\begin{aligned}
\dot{\mathbf{r}}_{i}= & \gamma\left(D_{0}-r_{i}\right) \mathbf{e}_{i}+2 \Gamma_{i} \mathbf{u}\left(\mathbf{r}_{i}\right) \\
& +\sum_{j} \Gamma_{j}\left\{\mathbf{u}\left(\mathbf{R}_{i j}+\frac{\mathbf{r}_{i}-\mathbf{r}_{j}}{2}\right)+\mathbf{u}\left(\mathbf{R}_{i j}+\frac{\mathbf{r}_{i}+\mathbf{r}_{j}}{2}\right)\right. \\
& \left.-\mathbf{u}\left(\mathbf{R}_{i j}-\frac{\mathbf{r}_{i}+\mathbf{r}_{j}}{2}\right)-\mathbf{u}\left(\mathbf{R}_{i j}-\frac{\mathbf{r}_{i}-\mathbf{r}_{j}}{2}\right)\right\}(\mathrm{A} 2)
\end{aligned}
$$

A Taylor expansion of the curled bracket yields

$$
\begin{aligned}
\dot{\mathbf{r}}_{i}= & \gamma\left(D_{0}-r_{i}\right) \mathbf{e}_{i}+2 \Gamma_{i} \mathbf{u}\left(\mathbf{r}_{i}\right) \\
& +\sum_{j} \Gamma_{j}\left\{\left(\mathbf{r}_{i}-\mathbf{r}_{j}\right) \cdot \nabla_{\mathbf{R}_{i j}}+\left(\mathbf{r}_{i}+\mathbf{r}_{j}\right) \cdot \nabla_{\mathbf{R}_{i j}}\right\} \mathbf{u}\left(\mathbf{R}_{i j}\right) \\
= & \gamma\left(D_{0}-r_{i}\right) \mathbf{e}_{i}+2 \Gamma_{i} \mathbf{u}\left(\mathbf{r}_{i}\right)+2 \sum_{j} \Gamma_{j} \mathbf{r}_{i} \cdot \nabla_{\mathbf{R}_{i j}} \mathbf{u}\left(\mathbf{R}_{i j}\right)
\end{aligned}
$$

where we have only retained the leading terms in $|\mathbf{r}| /|\mathbf{R}|$. The evolution equation for the center coordinate reads

$$
\begin{aligned}
\dot{\mathbf{R}}_{i}= & \frac{1}{2} \sum_{j} \Gamma_{j}\left\{\mathbf{u}\left(\mathbf{R}_{i j}+\frac{\mathbf{r}_{i}-\mathbf{r}_{j}}{2}\right)+\mathbf{u}\left(\mathbf{R}_{i j}+\frac{\mathbf{r}_{i}+\mathbf{r}_{j}}{2}\right)\right. \\
& \left.+\mathbf{u}\left(\mathbf{R}_{i j}-\frac{\mathbf{r}_{i}+\mathbf{r}_{j}}{2}\right)+\mathbf{u}\left(\mathbf{R}_{i j}-\frac{\mathbf{r}_{i}-\mathbf{r}_{j}}{2}\right)\right\}
\end{aligned}
$$


Again, a Taylor expansion yields

$$
\begin{aligned}
& \dot{\mathbf{R}}_{i}=2 \sum_{j} \Gamma_{j}\left[\mathbf{u}\left(\mathbf{R}_{i j}\right)\right. \\
& \left.+\frac{1}{8}\left\{\left[\left(\mathbf{r}_{i}-\mathbf{r}_{j}\right) \cdot \nabla_{\mathbf{R}_{i j}}\right]^{2}+\left[\left(\mathbf{r}_{i}+\mathbf{r}_{j}\right) \cdot \nabla_{\mathbf{R}_{i j}}\right]^{2}\right\} \mathbf{u}\left(\mathbf{R}_{i j}\right)\right] \\
& =2 \sum_{j} \Gamma_{j} \mathbf{u}\left(\mathbf{R}_{i j}\right) \\
& \quad+\frac{1}{4} \sum_{j} \Gamma_{j}\left[\left(\mathbf{r}_{i} \cdot \nabla_{\mathbf{R}_{i j}}\right)^{2}+\left(\mathbf{r}_{j} \cdot \nabla_{\mathbf{R}_{i j}}\right)^{2}\right] \mathbf{u}\left(\mathbf{R}_{i j}\right)
\end{aligned}
$$

The gradients of the velocity fields are now calculated according to

$$
\mathbf{r} \cdot \nabla_{\mathbf{R}} \frac{\mathbf{R}}{|\mathbf{R}|^{2}}=\frac{\mathbf{r}}{|\mathbf{R}|^{2}}-2 \frac{\mathbf{R}}{|\mathbf{R}|^{4}} \mathbf{r} \cdot \mathbf{R}
$$

which is needed in equation (35), and

$\left(\mathbf{r} \cdot \nabla_{\mathbf{R}}\right)^{2} \frac{\mathbf{R}}{|\mathbf{R}|^{2}}=-2 \frac{\mathbf{R}}{|\mathbf{R}|^{4}} \mathbf{r}^{2}-4 \frac{\mathbf{r}}{|\mathbf{R}|^{4}} \mathbf{r} \cdot \mathbf{R}+8 \frac{\mathbf{R}}{|\mathbf{R}|^{6}}(\mathbf{r} \cdot \mathbf{R})^{2}$

Now, this is the counterpart of equation (36).
[1] A.S. Monin, A. M. Yaglom, Statistical Fluid Mechanics, (Dover Publications, Mineola, 2007).

[2] U. Frisch, Turbulence: The Legacy of A.N. Kolmogorov, (Cambridge University Press, Cambridge 1995).

[3] A. Tsinober, An Informal Conceptual Introduction to Turbulence, (Springer Verlag Heidelberg, 2009).

[4] G. Falkovich, K. R. Sreenivasan, Phys. Today 59, 43 (2006).

[5] J. Cardy, G. Falkovich, K. Gawedzki, Non-equilibrium Statistical Mechanics and Turbulence (Cambridge University Press 2008).

[6] H. Helmholtz, Phil. Mag. (Ser 4) 33, 485 (1858); Phil. Mag. (Ser 4) 36, 337 (1868).

[7] G. R. Kirchhoff, Vorlesungen über mathematische Physik. Mechanik. (Teubner, Leibzig 1876).

[8] H. Aref, Ann. Rev. Fluid Mech. 15, 345 (1983).

[9] H. Aref, J. Math. Phys. 48, 065401 (2007).

[10] H. Aref, Fluid Dyn. Res. 39, 5 (2007).

[11] P. G. Saffman, Vortex dynamics (Cambridge University Press, 1992).

[12] P. K. Newton, The N-vortex problem (Springer-Verlag, New York, Berlin, Heidelberg, 2001).

[13] L. Onsager, Nuovo. Cim. Suppl. 6, 279 (1949).

[14] G. L. Eyink and K. R. Sreenivasan, Rev. Mod. Phys. 78, 87 (2006).

[15] G. Joyce and D. Montgomery, J. Plasma Phys. 10, 107 (1973).

[16] T. S. Lundgren and Y. B. Pointin, J. Stat. Phys. 17, 323 (1977).

[17] P. H. Chavanis, J. Stat. Mech. P05019 (2010).

[18] R. H. Kraichnan, Phys. Fluids 10, 1417 (1967).

[19] R. H. Kraichnan, J. Atmos. Sci. 33, 1521 (1926).

[20] S. Chen, R. E. Ecke, G.L. Eyink, M. Rivera, Minping Wan, and Z. Xian, Phys. Rev. Lett. 96, 084502 (2006).

[21] R. Benzi, M. Colella, M. Briscolini and P. Santangelo, Phys. Fluids A 4, 1036 (1992).
[22] J. B. Weiss and C. McWilliams, Phys. Fluids A 5, 608 (1993).

[23] E. D. Siggia and H. Aref, Phys. Fluids 24, 171 (1981).

[24] R. Friedrich, M. Voßkuhle, O. Kamps and M. Wilczek, Phys. Fluids 24, 125101 (2012).

[25] M. V. Melander, A. S. Styczek and N. J. Zabusky, Phys. Rev. Lett. 53, 1222 (1984).

[26] S. A. Balbus and J. F. Hawley, Astrophys. J. 376,214 (1991).

[27] K. Kleineberg and R. Friedrich, Phys. Rev. E 87, 033007 (2013).

[28] A. Vincent and M. Meneguzzi, J. Fluid Mech, 225:120 (1991)

[29] U. Frisch and P. L. Sulem, Phys. Fluids 27, 1911 (1984).

[30] L. Smith and V. Yakhot, Phys. Rev. Lett. 71, 352 (1993).

[31] G. Boffetta, A. Celani and M. Vergassola, Phys. Rev. E 61, R 29 (2000).

[32] J. Paret and P. Tabeling, Phys. Rev. Lett. 79, 4162 (1997).

[33] J. Paret and P. Tabeling, Phys. Fluids 10, 3126 (1998)

[34] M. Toda, R. Kubo and N. Saitô, Statistical Physics I (Springer-Verlag New York, Tokyo, Berlin, Heidelberg, 1983).

[35] I. M. Lifshitz and V. V. Slyozov, J. Phys. Cem. Solids 19 (1961).

[36] G. L. Eyink, J. Fluid Mech. 549, 191 (2006).

[37] M. Wilczek and R. Friedrich, Phys. Rev. E 80, 016316 (2009).

[38] M. Wilczek, A. Daitche and R. Friedrich, EPL, 9334003 (2011).

[39] M. Wilczek, A. Daitche and R. Friedrich, J. Fluid Mech. 676, 191 (2011).

[40] J. Lülff, M. Wilczek and R. Friedrich, New J. Phys. 13, 015002 (2011). 